\title{
Optical vortices with starlight
}

\author{
G. Anzolin ${ }^{1,2}$, F. Tamburini ${ }^{1}$, A. Bianchini ${ }^{1}$, G. Umbriaco ${ }^{1}$, and C. Barbieri ${ }^{1}$ \\ 1 Dipartimento di Astronomia, Università di Padova, vicolo dell'Osservatorio 3, 35122 Padova, Italy \\ e-mail: gabriele.anzolin@unipd.it \\ 2 INAF-Osservatorio Astronomico di Capodimonte, salita Moiariello 16, 80131 Napoli, Italy
}

Received 26 June 2008 / Accepted 18 July 2008

\section{ABSTRACT}

\begin{abstract}
Aims. In this paper we present our first observations at the Asiago $122 \mathrm{~cm}$ telescope of $\ell=1$ optical vortices generated with starlight beams.

Methods. We used a fork-hologram blazed at the first diffraction order as a phase modifying device. The multiple system Rasalgethi ( $\alpha$ Herculis) in white light and the single star Arcturus ( $\alpha$ Bootis) through a $300 \AA$ bandpass were observed using a fast CCD camera. In the first case we could adopt the Lucky Imaging approach to partially correct for seeing effects.

Results. For both stars, the optical vortices could be clearly detected above the smearing caused by the mediocre seeing conditions. The profiles of the optical vortices produced by the beams of the two main components of the $\alpha$ Her system are consistent with numerically simulated on-axis and off-axis optical vortices. The optical vortices produced by $\alpha$ Boo can also be reproduced by numerical simulations. Our experiments confirm that the ratio between the intensity peaks of an optical vortex can be extremely sensitive to off-axis displacements of the beam.

Conclusions. Our results give insights for future astronomical applications of optical vortices both for space telescopes and groundbased telescopes with good seeing conditions and adaptive optics devices. The properties of optical vortices can be used to perform high precision astrometry and tip/tilt correction of the isoplanatic field. We are now designing a $\ell=2$ optical vortex coronagraph around a continuous spiral phase plate. We also point out that optical vortices could find extremely interesting applications also in the infrared and radio wavelengths.
\end{abstract}

Key words. instrumentation: miscellaneous - techniques: high angular resolution - atmospheric effects

\section{Introduction}

During the past decade, light has been shown to carry more information than what was commonly believed: a new degree of freedom has been identified in the orbital angular momentum (OAM, Allen et al. 1992), which is associated to the spatial intensity distribution of an optical beam. OAM is a property of light beams housing phase defects called optical vortices (OVs, Coullet et al. 1989). In such beams, the wavefront has a helicoidal shape, causing the Poynting vector to spin as a corkscrew around the direction of propagation (Padgett \& Allen 1995).

Singular beams can be mathematically described by a superposition of Laguerre-Gaussian (L-G) modes characterized by the two integer-valued indices $\ell$ and $p$. The azimuthal index $\ell$ describes the number of twists of the helical wavefront and the radial index $p$ gives the number of radial nodes of the mode. The electromagnetic field amplitude of a generic L-G mode in a plane orthogonal to the direction of propagation $z$ (that is also the symmetry axis of the beam) is

$u_{\ell p}(r, \theta) \propto\left(\frac{r \sqrt{2}}{w}\right)^{|\ell|} L_{p}^{|\ell|}\left(\frac{2 r^{2}}{w^{2}}\right) \exp \left(-\frac{r^{2}}{w^{2}}\right) \exp (-\mathrm{i} \ell \theta)$

where $w$ is a scale parameter and $L_{n}^{m}(x)$ is the associated Laguerre polynomial. The presence of a phase factor $\exp (-\mathrm{i} \ell \theta)$ implies that these cylindrically symmetrical modes carry an OAM equal to $\ell \hbar$ per photon, relative to their symmetry axis. For the same reason, a phase singularity is embedded in the wavefront, all along the propagation axis, with topological charge equal to $\ell$. In other words, the phase of the beam increases of a quantity $\ell 2 \pi$ over a circular path closed around the $z$ axis. The intensity distribution of an L-G mode with $p=0$ and $\ell \neq 0$ is generally shaped as a ring with a central dark hole, where the intensity is null due to total destructive interference.

Experimentally, OVs can be generated by using a phase modifying device (PMD) that imprints a certain vorticity on the phase distribution of the original beam. This technique has allowed to apply OVs in diverse research fields such as laboratory optics, nanotechnologies and biology (see e.g. Grier 2003; Molina-Terriza et al. 2007). Optical singularities have been detected also in beams propagating through nonlinear optical systems (Arecchi et al. 1991) and Kerr nonlinear refractive media (Swartzlander \& Law 1992). This led to the argument that OVs could be naturally generated by some astrophysical environments, possibly related to turbulent interstellar media with density discontinuities on wide scale ranges or to the distorted geometry around Kerr black holes (Harwit 2003).

Recently, the properties of beams carrying OVs have attracted attention for practical astronomical applications:

1. overcoming the Rayleigh separability criterion by direct imaging and analysis of tiny deviations in the intensity patterns of superposed OVs produced by very close point sources (Tamburini et al. 2006a,b; Barbieri et al. 2007);

2. improving the capability of direct observation of extrasolar planets by "peering into the darkness" of an OV generated by a PMD inserted in the optical path of a Lyot coronagraph (Swartzlander 2001; Foo et al. 2005; Lee et al. 2006).

The former application is better achievable with $\ell=1 \mathrm{OVs}$, while in the second case only evenly-charged OVs generated by 
a perfect Airy diffraction pattern ensure the total rejection of the light of the on-axis source (Mawet et al. 2005). Indeed, these astronomical applications would give their best performances with instruments placed outside the atmosphere. Ground-based telescopes, instead, will always feel the detrimental effects of atmospheric turbulence, even with adaptive optics. Therefore, it is important to know how the seeing can affect the pattern of OVs generated by stellar sources. A first investigation in this field has been done by Jenkins (2008) in a paper concerning the coronagraphy, in which it was analytically demonstrated that an OV coronagraph can give good performances also in ground-based telescopes operating with a partial correction of the atmospheric turbulence. The feasibility of ground-based $\ell=2 \mathrm{OV}$ coronagraphy has been demonstrated very recently by Swartzlander et al. (2008).

In this Paper we present the results of the direct observation of OVs generated with starlight beams at the Asiago $122 \mathrm{~cm}$ telescope. In Sect. 2 we describe the most efficient PMDs. In Sect. 3 we discuss the expected pattern of OV in ground-based telescopes. In Sect. 4 we report our observations and data reduction. In Sects. 5 and 6 we discuss our results, draw some conclusions and plans for future activity.

\section{Phase modifying devices}

A PMD is an optical element characterized by a central optical singularity which is responsible for changing the phase structure of an incoming beam. If an axially symmetrical monochromatic beam intersects a PMD on-axis, i.e. the symmetry axis of the beam intersects the optical singularity of the PMD, then the pattern of the OV presents a circular symmetry with a central dark region (a "donut" shape). Instead, if the beam is shifted off-axis, the dark region appears displaced off center and the OV loses its circular symmetry (Vaziri et al. 2002).

The most efficient PMDs are computer-generated forkholograms (Bazhenov et al. 1990) and spiral phase plates (SPPs, Beijersbergen et al. 1994). SPPs are helicoidal transmission optical devices with a given total thickness variation $h_{\mathrm{s}}$. The topological charge $\ell$ imposed to a monochromatic on-axis beam is $\ell=\Delta n(\lambda) h_{\mathrm{s}} / \lambda$, where $\lambda$ is the wavelength and $\Delta n$ is the difference between the refraction indices of the SPP material and the surrounding medium. Instead, a fork-hologram is an optical device which resemble a grating with a number $l$ of dislocations on its center. A fork-hologram can be blazed in order to improve the diffraction efficiency in a specific diffraction order. In this case, the transmission function in polar coordinates can be written as

$T(r, \phi)=\exp \left[\mathrm{i} \frac{\delta}{2 \pi} \bmod \left(l \phi-\frac{2 \pi}{\Lambda} r \cos \phi, 2 \pi\right)\right]$

where $\delta$ is the amplitude of the phase modulation ${ }^{1}, \Lambda$ is the spatial period of the grooves away from the center and $\bmod (a, b)=$ $a-b \operatorname{int}(a / b)$. When a monochromatic beam intersects a forkhologram exactly on-axis, it produces OVs characterized by the value of the integer charge $\ell=m l$, where $m$ is the diffraction order. The contribution of higher-order modes is negligible, since fork-holograms produce OVs with a mode purity up to $90 \%$ (Clifford et al. 1998).

If we are not using monochromatic light, which is usually preferable while observing faint stellar objects, the "donut"shaped structure of the observed OVs will be further modified (Palacios et al. 2004; Shvedov et al. 2005). Using SPPs, all OVs

\footnotetext{
${ }^{1} \delta=2 \pi$ for a fork-hologram blazed at the first diffraction order.
}

have the same axis of symmetry but present different topological charges as a function of $\lambda$. Thus, the transmitted beam is composed of a superposition of coaxial "donuts" of different sizes. As a result, the OV will present a smoother profile with a partial filling of the central dark region. Swartzlander (2006) proposed an optical solution to correct this effect, but achromaticity was found only for a limited bandwidth $(\sim 1000 \AA)$ in the visible. Differently from SPPs, fork-holograms present the advantage of generating OVs with the same $\ell$ at all wavelengths. Each spectral component will be instead dispersed at a different angle according to the usual grating equation. Therefore, the intensity pattern of a non-monochromatic OV at the first diffraction order will appear as a ring stretched along the direction of the dispersion with a central dark strip.

In our experiments at the telescope we decided to use an $l=1$ fork-hologram blazed at the first diffraction order to be able to simultaneously observe both the stellar speckle image, at the zero-th diffraction order, and the OV at the first diffraction order. The choice of $l=1$ avoided some possible experimental complications as high topological charge OVs might become unstable within the instrumental optical path and split in singlecharged OVs (Nye \& Berry 1974).

\section{Optical vortices with ground-based telescopes}

The pattern of an OV generated with a PMD placed near the focal plane of a telescope strongly depends on the spatial intensity distribution of the stellar beam, i.e. the point spread function (PSF) observed.

Diffraction images generated by point-like stellar sources on the focal plane of a diffraction-limited telescope present PSFs that are described by the Airy function $I_{\mathrm{A}} \propto\left[J_{1}(r) / r\right]^{2}$, where $J_{1}(x)$ is the Bessel function of the first kind and $r=k A \sin \theta$ ( $k=2 \pi / \lambda$ and $A$ is the aperture radius of the telescope). Instead, for an uncompensated ground-based telescope, the PSF of a stellar source is degraded by the atmospheric turbulence and consists in a central nearly-Gaussian core with a radius determined by the seeing and an outer exponentially decreasing halo (King 1971). When the stellar PSFs produced in these two limiting conditions are exactly set on-axis of the PMD, OVs with same integer topological charge are generated. An OV produced by a monochromatic Gaussian beam (hereafter "Gaussian OV"), like those produced by the seeing, can be well described by an L-G mode (Vaziri et al. 2002). This is quite different from the OV produced by a diffraction-limited beam (hereafter "Airy OV"), the analytical expression of which is given in Mawet et al. (2005). As an useful example, Fig. 1 shows the numerical simulations of the two types of single-charged monochromatic OVs having the same maximum intensity radius $r_{\max }$, together with their radial profiles. In both cases the phase singularity produces a null intensity at the center. The main difference between the two types of OV resides in the shape of the intensity maxima: for an "Airy OV" they appear sharp and narrow, whereas those of a "Gaussian OV" are smooth and broad. OVs with higher values of the topological charge also share this general characteristics, with the additional condition that even-valued "Airy OVs" have zero intensity for any $r<r_{\max }$. For this reason they are used for coronagraphy. We recall that, even when using slightly converging stellar beams like those produced by telescopes with high focal ratios, the structure of the OVs are not significantly changed (Bekshaev \& Karamoch 2008).

Another important task in the cited astronomical applications of OVs consists in keeping a stellar PSF exactly on-axis of the 


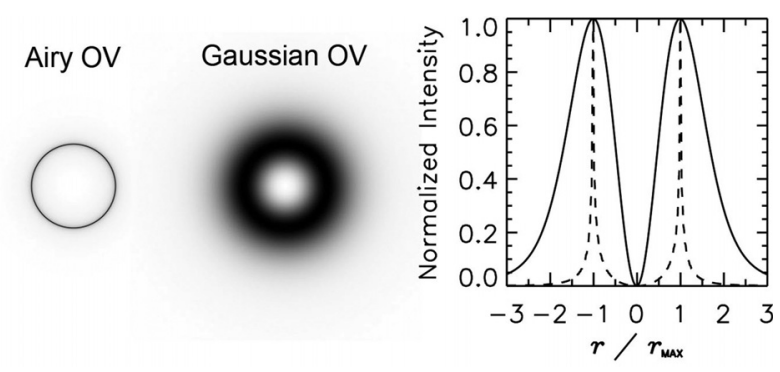

Fig. 1. Numerically simulated intensity distributions of $\ell=1$ monochromatic on-axis OVs generated with an Airy pattern (left) and with a Gaussian beam (center). The input beams was chosen in order to produce OVs with the same maximum intensity radius $r_{\max }$. Right panel: radial profiles of the two OVs. The solid line represents the "Gaussian OV", while the dashed line represents the "Airy OV". The intensity scale of the two profiles has been normalized to the correspondent maximum value.

PMD for all the duration of the observation. In fact, the atmospheric turbulence causes the stellar beam to wander around the line of sight. Therefore, we need to know the actual position of the stellar image in the focal plane of the telescope where the PMD is placed. In this work, we tried to solve this problem by using the Lucky Imaging (Law et al. 2006a,b) approach. The Lucky Imaging is one of the speckle imaging techniques used as alternative to adaptive optics. It basically consists in collecting a large number of images with exposure times shorter than the turbulence timescale ( $\sim 10-100 \mathrm{~ms}$ in the optical/near-infrared). A single nearly diffraction-limited image can occasionally be produced when most of the stellar light falls in a single bright speckle. Such "lucky exposures" (Fried 1978) can be selected and, then, properly combined to produce a high-quality image. We have here adopted the obvious additional criterion of selecting only those exposures where the target was centered with the PMD.

\section{Observations and data reduction}

Observations were carried out in May 2005 with the Asiago $122 \mathrm{~cm}$ telescope. Our targets were the multiple system Rasalgethi ( $\alpha$ Her) and the single star Arcturus ( $\alpha$ Boo). $\alpha$ Her is a visual binary composed by two spectroscopic binary systems which are presently separated by 4.'7: $\alpha$ Her A, formed by an M5 Ib-II semiregular variable $(V=2.7-4.0)$ plus a fainter companion separated by 0.'19 (McAlister et al. 1989), and $\alpha$ Her B, containing a G0 II-III giant $(V=5.4)$ and a secondary which separation was spectroscopically estimated as 0.'0035 (Halbwachs 1981). $\alpha$ Boo is a single star with visual magnitude $V=0.04$ and spectral type K1.5 III.

In our observations we used the two different optical setups sketched in Fig. 2. The $l=1$ fork-hologram $H$ was obtained from a quartz plate by means of electron beam lithography. It has 38.5 grooves $\mathrm{mm}^{-1}$ (spatial period $\Lambda=26 \mu \mathrm{m}$ ) and an active area of $2.6 \times 2.6 \mathrm{~mm}^{2}$. Due to technical limitations, the depth of the grooves was subdivided in 8 discrete levels. The forkhologram is blazed at the first diffraction order with a diffraction efficiency of $\sim 80 \%$ at $702 \mathrm{~nm}$.

We used a fast CCD camera with $660 \times 494$ pixels $(7.4 \times$ $\left.7.4 \mu \mathrm{m}^{2}\right), 16$ bit dynamical range and spectral response ranging from 4000 to $6700 \AA$ peaked at $5200 \AA$, which allowed to partially correct the stellar PSF for the seeing effects.

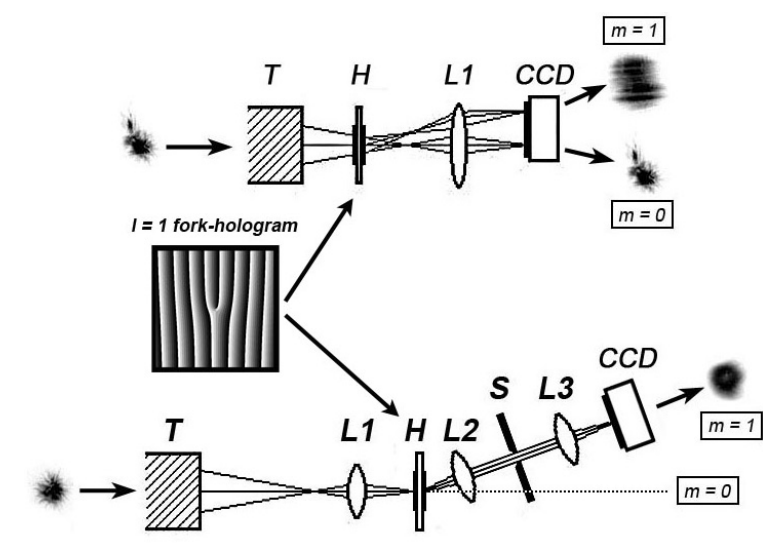

Fig. 2. Optical setups, without (top) and with (bottom) spatial filter. $T$ is the telescope; $L 1, L 2, L 3$ are lenses; $H$ is the $l=1$ fork-hologram (its pattern is depicted in the middle of the figure); $S$ is the slit. Stellar speckle patterns are sketched on the left of the optical setups, while the output images at the $m=0$ and $m=1$ diffraction order are on the right. All angles and displacements are exaggerated for clarity.

To select the "best" frames we proceeded as follows:

1. when the zero-th diffraction order was visible, we determined the full width at half maximum (FWHM) of the target PSF, after the subtraction of the mean sky level, and then selected the best $10 \%$ frames;

2. we further selected the frames in which the target was also on-axis by analyzing the intensity profiles of the observed OVs at the first diffraction order;

The mechanical design of the lower setup of Fig. 2 prevented the observation of the zero-th diffraction order. In this case, we could only select those frames in which the observed OVs did present a symmetrical structure indicating that the target beam crossed the center of the fork-hologram.

\subsection{First experiment: non-monochromatic optical vortices}

We observed $\alpha$ Her in non-monochromatic light with the upper optical setup of Fig. 2 that allowed to simultaneously see the zero-th and the first diffraction order. The fork-hologram was placed $30 \mathrm{~mm}$ before the F/16 Cassegrain focal plane of the telescope as we wished to achieve two conditions:

- to obtain well separated OV patterns on the focal plane of lens $L 1$ (this was however granted by the angular separation of the stars);

- to have both light beams large enough to cross a significant area of the fork-hologram and, in particular, the central singularity.

We set $\alpha$ Her A at the center of the optical system and, then, recorded a sequence of 860 frames at a time step of $70 \mathrm{~ms}$. To select the best exposures, we used the PSF of the unsaturated $\alpha$ Her B component and found that $10 \%$ of the frames presented a FWHM below $\sim 1$ '. 6 . The on-axis condition for $\alpha$ Her A was further recognized when the peaks of the corresponding dispersed OV had the same intensity within the experimental errors. The images and the chromatically dispersed OVs resulting from the summation of all the best frames selected are shown in Fig. 3. The average radial profile of the PSF of $\alpha$ Her B (see Fig. 4) is constituted by a Gaussian core superposed to a halo that we fitted with another coaxial Gaussian, for simplicity. The FWHM 


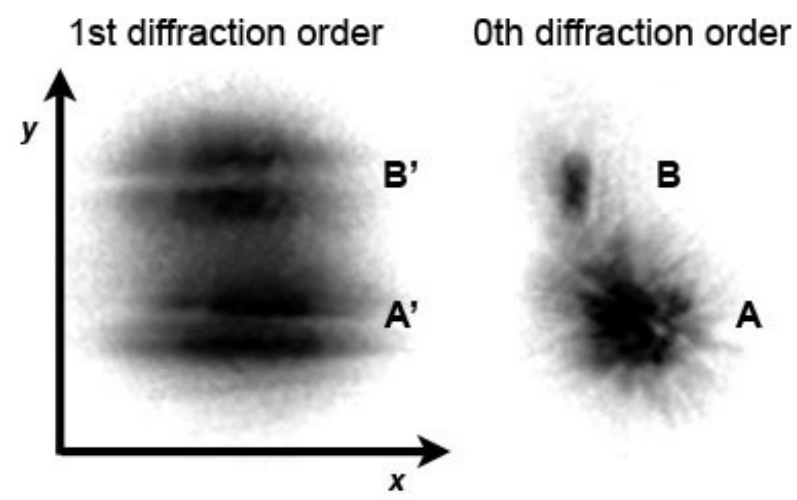

Fig. 3. Image (right) and dispersed OVs (left) of $\alpha$ Her A and B obtained by adding the selected best frames (see text). Intensities are displayed in a squared greyscale. The OV profiles were taken along the $y$ axis, perpendicular to the direction of dispersion $x$.

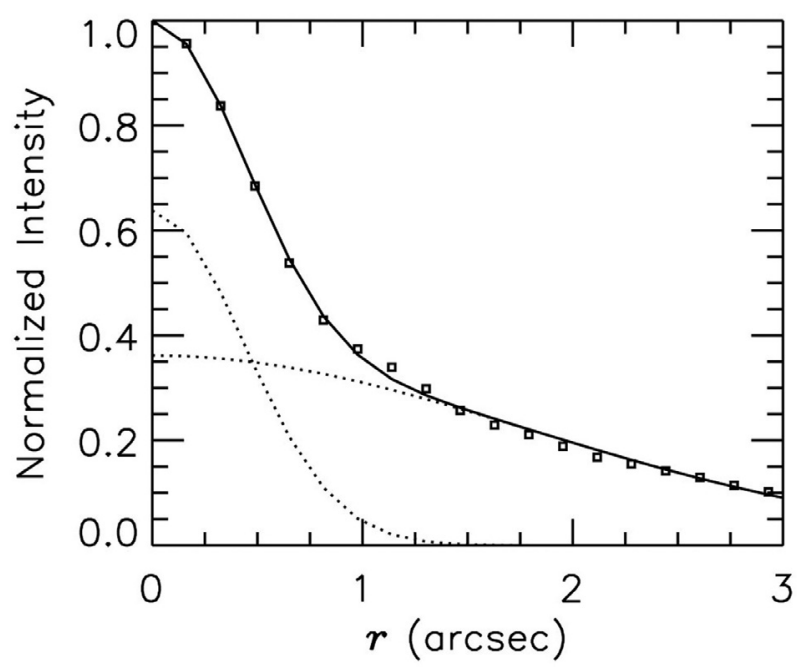

Fig. 4. Radially averaged profile of the PSF of $\alpha$ Her B obtained by adding the selected best frames (open squares). The solid line represents the best-fit model constituted by two superposed coaxial Gaussians. The two Gaussians components are shown separately (dotted lines), for clarity.

of the two Gaussians are 1" and 4.'2 respectively, the latter being roughly consistent with the seeing. Figure 5 shows the mean profiles of the OVs obtained by averaging 10 pixels wide strips extracted perpendicularly to the dispersion.

\subsection{Second experiment: narrow-band optical vortices}

The observations of the single star $\alpha$ Boo were performed with the optical setup shown in the lower part of Fig. 2. The forkhologram was placed at the focus of lens $L 1$ where the scale of the telescope was magnified by a factor $\sim 1.5$. Thus, the FWHM of the PSF of the $\sim 3^{\prime \prime}$ seeing image was $0.5 \mathrm{~mm}$. We introduced the spatial filter $S$ made of a $0.1 \mathrm{~mm}$ slit placed in the Fourier plane of the collimating lens $L 2$. The slit essentially acts as a tunable bandpass filter with a flat spectral response. Therefore, by limiting the spectral range to $\sim 300 \AA$ in the visible we could somehow restore the "donut" shape of a monochromatic OV (Leach \& Padgett 2003; Tamburini et al. 2006a), still ensuring a reasonable signal-to-noise $(\mathrm{S} / \mathrm{N})$ ratio. We obtained a sequence of 890 frames with exposure times of $70 \mathrm{~ms}$. Due to the unobservability of the zero-th order, we could only look for on-axis

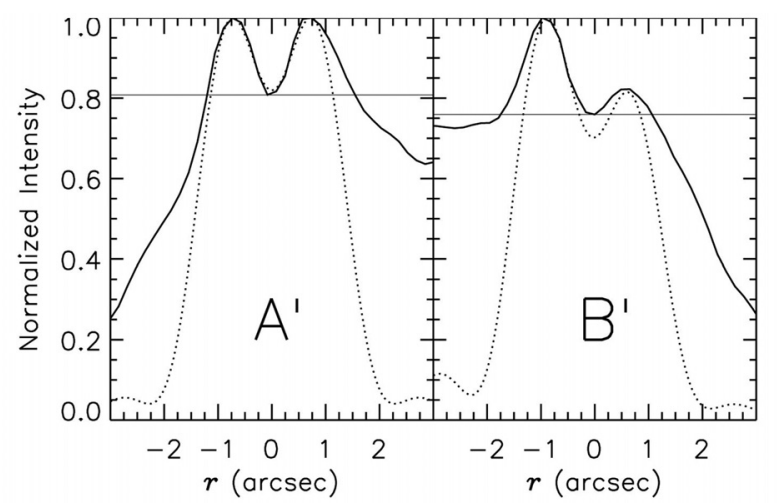

Fig. 5. Profiles of the OVs generated by $\alpha$ Her A $\left(A^{\prime}\right.$, left panel $)$ and $\alpha$ Her B ( $B^{\prime}$, right panel) extracted along the $y$ axis of Fig. 3. Dotted lines represent the numerical simulations of $\ell=1$ chromatically dispersed OVs generated by a pupil with a circular $7 \%$ obstruction. Thin solid lines indicate the intensity value at the central dips of the observed OVs.

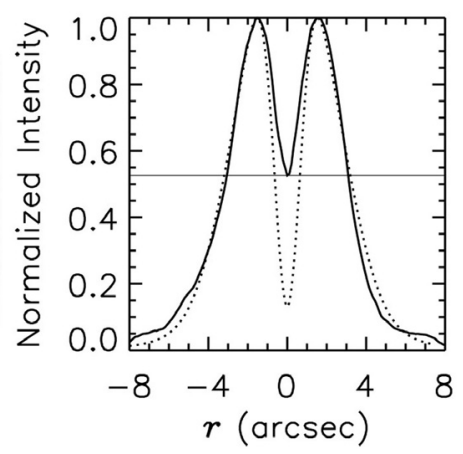

Fig. 6. Left: the narrow-band $\mathrm{OV}$ of $\alpha$ Boo obtained by summing the selected frames (see text). The intensity is displayed in a squared greyscale. Right: profile of the OV across the direction perpendicular to the dispersion (solid line). The dotted line represents the numerical simulation of an $\ell=1 \mathrm{OV}$ produced by a PSF modelled as described in the text, with a spectral range of $300 \AA$. The thin solid line indicates the observed intensity in the central dark region.

condition of $\alpha$ Boo by analyzing the intensity profiles of the OV. We then choose those frames in which the ratios of the intensity peaks measured along two perpendicular axis across the OV were close to unity within $1 \%$ errors. In this way, we can reveal off-axis displacements along any radial direction. The mean OV pattern obtained by adding all the selected frames is shown in the left part of Fig. 6 .

\section{Results and discussion}

\subsection{Non-monochromatic optical vortices}

To check the results of the first experiment, we performed numerical simulations of non-monochromatic OVs produced with an $l=1$ fork-hologram. Since we set our fork-hologram in an intrafocal position, the image of the stellar beam did approximately coincide with that of the pupil. In our simulation we used a simplified image model made of an uniformly illuminated disk with radius $a$ plus a central circular $7 \%$ obstruction, like that produced by the secondary mirror of the $122 \mathrm{~cm}$ Asiago telescope. To simulate the observed OV profile $A^{\prime}$ of Fig. 5 we assumed a perfectly on-axis pupil image, while the $B^{\prime}$ profile was reproduced by imposing an off-axis relative displacement 
$\rho / a=0.31, \rho$ being the linear separation of the two stars on the fork-hologram plane. For the off-axis OV we had also to consider that the displacement vector (the line joining $\alpha$ Her A and B) was tilted by an angle of $30^{\circ}$ with respect to the dispersion. The intensity profiles of the simulated OVs are shown in Fig. 5. Both the observed $A^{\prime}$ and $B^{\prime}$ profiles show extended smooth wings not properly reproduced by our simplified simulations. This is probably due to the insufficiently short exposures which did not allow a proper compensation of the seeing effects. However, the most remarkable result concerns the ratio $R$ of the two intensity maxima. By fitting with Gaussians the two peaks of the observed $A^{\prime}$ and $B^{\prime}$ profiles, we measured $R_{A^{\prime}}=0.995 \pm 0.005$ and $R_{B^{\prime}}=0.83 \pm 0.01$, respectively. These values are in good agreement with the values 1.0 and 0.82 predicted by our numerical simulations. We notice that the central dip in $A^{\prime}$ is 0.81 times the maximum intensity, still in agreement with the predicted value of 0.82 . For $B^{\prime}$, instead, the dip is 0.76 , while the numerical simulation predicts 0.70 .

\subsection{Narrow-band optical vortices}

In the second experiment we observed a single star focused at the center of the fork-hologram. Since in this case the zero-th diffraction order was not visible, we could not perform Lucky Imaging and the best $17 \%$ images were selected only on the basis of the circular symmetry of the OV. Using a spatial filter to limit the wavelength range, we obtained a "donut"-shaped OV similar to the "Gaussian OV" shown in Fig. 1.

Also in this case we simulated the OV pattern by assuming a PSF modelled with two Gaussians. Here we modelled the effects of the dispersion over a spectral range of $300 \AA$. We find that the OV which best-fits the observed profile is produced by a stellar PSF composed by a dominant core with a FWHM of 3.' 6 and a fainter halo with $\sim 7$ " FWHM, its peak value being only $2 \%$ that of the central Gaussian. The simulated OV quite remarkably reproduces the wings of the observed OV, but not so well the central dip which should be $13 \%$ the intensity of the two maxima, while it is observed at the $52 \%$ level (see the right panel of Fig. 6). This effect is very likely due to the loss of the starlight coherence produced by the bad seeing conditions plus our relatively long exposure times.

Intensity inhomogeneities in the observed "donut" pattern can be ascribed to the presence of dust grains in the optical path and/or construction imperfections of the fork-hologram.

\subsection{The relation between the maxima intensity ratio and the off-axis position}

The results of our experiments represent a first test bench to relate the intensity profiles of the observed OVs to the offaxis position of the starlight beam on the fork-hologram. In fact, the intensity peaks extracted along any direction across on-axis OVs are equal, while the intensity profiles of off-axis OVs become asymmetric along the direction of the displacement. The ratio $R$ between the two intensity peaks is then a function of the off-axis displacement $\rho$ on the hologram plane. For a monochromatic Gaussian beam, this relation can be analytically derived, as detailed in Appendix A. For other types of beams, e.g. non-Gaussian and/or non-monochromatic, $R$ can be numerically evaluated.

We have performed numerical simulations of OVs produced by beams with various intensity distributions, namely a Gaussian, two Gaussians (like in our second experiment) and

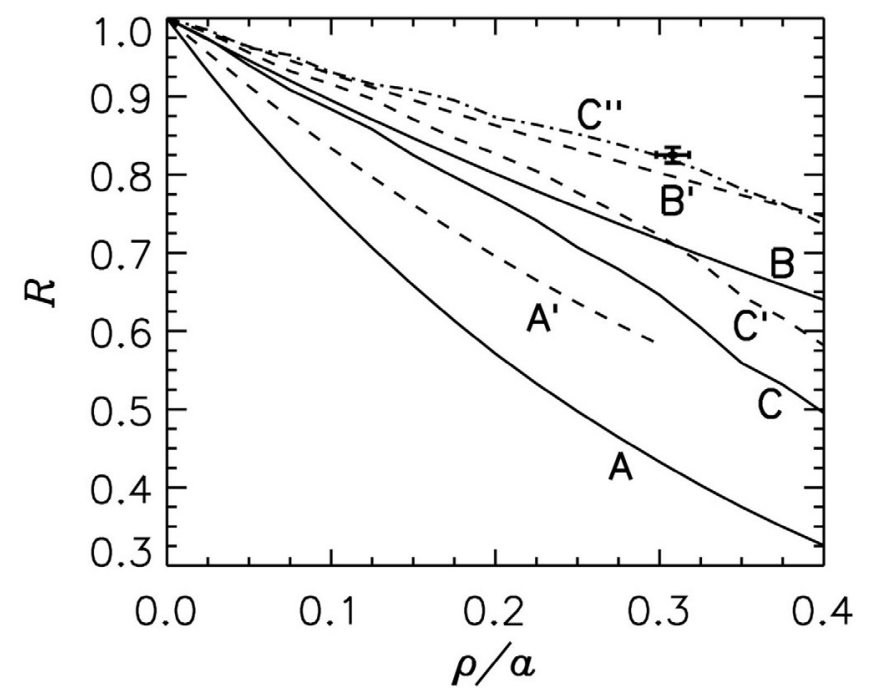

Fig. 7. Ratios of the intensity maxima plotted as a function of the offaxis relative position $\rho / a$ for $\ell=1$ OVs generated by: (A) a monochromatic Gaussian beam; (A') a non-monochromatic Gaussian beam; (B) two monochromatic coaxial Gaussians; ( $\left.\mathrm{B}^{\prime}\right)$ two non-monochromatic coaxial Gaussians; $(\mathrm{C})$ a pupil in monochromatic light; $\left(\mathrm{C}^{\prime}\right)$ a pupil in non-monochromatic light; $\left(\mathrm{C}^{\prime \prime}\right)$ a pupil in non-monochromatic light where the off-axis displacement is inclined by $30^{\circ}$ with respect to the dispersion. The pupil model adopted in these simulations is described in Sect. 5.1. The experimental result obtained from the analysis of the OV generated by $\alpha$ Her B is indicated by the black dot (error bars are at the $1 \sigma$ confidence level).

a uniform pupil with a $7 \%$ central obstruction (like in our first experiment). We have then calculated $R$ for different values of the off-axis relative position $\rho / a$, where $2 a$ is the FWHM of the intensity distribution in case of Gaussian beams or the outer diameter in case of the pupil image. The results of our simulations are shown in Fig. 7, for both monochromatic and nonmonochromatic light. For a non-monochromatic Gaussian beam, $R$ cannot be evaluated above $\rho / a=0.3$ because the fainter peak becomes indistinguishable. As we can see, $R$ decreases as the off-axis displacement increases for all the beams considered. This is the result of the off-axis migration of the central dark region. $R$ decreases more rapidly for Gaussian OVs and, in general, for monochromatic light. The experimental result obtained from the analysis of the OV generated by $\alpha$ Her B is also reported in Fig. 7. Our measure fits quite well the theoretical curve $\mathrm{C}^{\prime \prime}$ obtained for an obstructed pupil displaced off-axis along a direction inclined by $30^{\circ}$ with respect to the dispersion of the fork-hologram.

The capability of detecting small off-axis displacements depends on the precision of the measure of $R$. However, the calibration of the relation $\rho / a$ vs. $R$ requires the knowledge of the beam structure, i.e. the profile of its intensity distribution. When the star is focused on the fork-hologram, $2 a$ roughly corresponds to the FWHM of the PSF. This means that the maximum sensitivity can be achieved with nearly diffraction limited stellar images. For this reason, Lucky Imaging or adaptive optics would provide the best results.

As an example, in our experiments with relatively bright stars we typically had $S / N \gtrsim 100$ and an error of $\sim 1 \%$ associated to the measure of $R$. Assuming a two Gaussians PSF, like that adopted to fit the OV observed in our second experiment, the smallest off-axis relative displacement inferred by Fig. 7 is $\rho / a \sim 0.01$. Since the assumed PSF corresponded to $a=1 . \prime 8$, the minimum angular displacement would be 0.02 . We notice 
that this value is comparable to the astrometric precision of $0 .^{\prime} 01$ obtained by fitting a Gaussian to the core of the PSF of $\alpha$ Her B. Figure 7 confirms that a higher precision is achievable when the PSF is modelled with a single Gaussian. In that case, still assuming a $1 \%$ error on $R$, the minimum off-axis relative displacement would be $\rho / a=0.004$, for monochromatic beams, or $\rho / a=0.006$, for non-monochromatic light.

\section{Conclusions}

In this paper we have presented and analyzed OVs generated with starlight beams crossing an $l=1$ fork-hologram placed near the F/16 Cassegrain focus of the Asiago $122 \mathrm{~cm}$ telescope. Our observations were carried on under mediocre seeing conditions $\left(3^{\prime \prime}-3^{\prime \prime} 8\right)$.

The two main components of the $\alpha$ Her system were observed in white light adopting the Lucky Imaging approach, which allowed to partially overcome the effects of the seeing. In this first experiment we used large beams (fork-hologram in intrafocal position) in order to test the sensitivity of the peaks intensity ratio $R$ for small relative off-axis displacements $\rho / a$. The OV profiles produced by the on-axis and the off-axis components were consistent with our numerical simulations.

In the second experiment we observed the single star $\alpha$ Boo and demonstrated that, by selecting a bandpass width of $\sim 300 \AA$ in the visible, we can obtain a rather symmetrical on-axis OV, very similar to the monochromatic one. The OV pattern was however affected by a partial filling of the central dark region due to residual chromaticity and the lack of light coherence produced by the atmospheric turbulence. Considering that the error associated to peaks intensity ratio $R$ was $\sim 1 \%$, we infer that, under those observational conditions, the minimum angular displacement detectable would have been 0 .' $^{\prime} 02$.

We numerically simulated OVs produced by beams with different intensity distributions and showed how $R$ decreases as $\rho / a$ increases for all the cases considered (see Fig. 7). We also found that $R$ may become extremely sensitive to the off-axis displacement of the beam, in particular for a Gaussian beam. In a previous paper (Tamburini et al. 2006a) we used the asymmetries observed in the intensity profiles of $\ell=1 \mathrm{OV}$ s to detect the presence an off-axis companion. We demonstrated that this technique could overcome the standard resolving power of a telescope. The results of this paper suggest that the OV generated by a single star can be also used to perform astrometry. Since $\rho / a$ increases as $a$ decreases, it is evident that under good seeing conditions or with nearly diffraction limited stellar images the astrometric precision achievable could be competitive to standard PSF astrometry.

To perform OV coronagraphy, "Airy OVs" with even values of their topological charge are required because diffraction limited beams produce larger central dark regions with null intensity. Quite recently, Swartzlander et al. (2008) performed OV coronagraphy with a $20 \mathrm{~cm}$ ground-based telescope using a simple adaptive optics module. On the basis of our results, we are also projecting an OV coronagraph with a PMD made by an $\ell=2$ SPP to be mounted at the Asiago telescope. This will represent a further test bench for future applications in space telescopes, where OV coronagraphy would provide the best results.

We finally suggest that a PMD placed within an adaptive optics system could be used to improve the tip/tilt correction of the wavefront for a small field of view. We might in fact correct the isokinetic patch the size of which, like the isoplanatic field, strongly depends on the seeing and $\lambda$. This method might then offer interesting applications in the far infrared and radio wavelengths. Actually, an application of phase singularities of the electromagnetic field and their use in the implementation of a coronagraph in the radio domain was suggested by Thidé et al. (2007).

Acknowledgements. We would like to thank the Institut für Experimentalphysik, University of Wien (Zeilinger-Gruppe) for support, helpful discussions and comments. We also thank professor Bo Thidé for helpful comments. This work has been partially supported by the University of Padova, by the Ministry of University and Research and by the CARIPARO Foundation inside the 2006 Program of Excellence.

\section{Appendix A: Properties of "Gaussian optical vortices"}

Consider a monochromatic Gaussian beam with an intensity distribution $I_{\mathrm{G}}(\rho, \phi) \propto \exp \left(-\rho^{2} / w_{0}^{2}\right)$. Here, the parameter $w_{0}$ roughly represents the radius of the beam, as the FWHM of the Gaussian is $2 \sqrt{\ln 2} w_{0}$.

The amplitude distribution of an OV generated at the first diffraction order of an $l=1$ fork-hologram can be expressed, in paraxial conditions, as a coherent superposition of L-G modes with topological charge $\ell=1$ and $\ell=0$ (Vaziri et al. 2002). In a plane $(r, \theta)$ perpendicular to the direction $z^{\prime}$ of the first diffraction order the amplitude distribution is then

$u_{\text {out }}(r, \theta)=\frac{1}{\sqrt{1+\gamma^{2}}}\left[u_{00}(r, \theta)+\gamma u_{10}(r, \theta)\right]$

where $\gamma$ is a weighting parameter. The topological charge of the output beam is $\ell_{\text {out }}=\sum_{\ell} \sum_{p} \ell\left|C_{\ell p}\right|^{2}$, where $C_{\ell p}=$ $\int_{0}^{2 \pi} \int_{0}^{\infty} u_{\text {out }} u_{\ell p}^{*} r \mathrm{~d} r \mathrm{~d} \theta$ and can assume any value in the interval $0 \leq \ell_{\text {out }} \leq 1$. We see that $\gamma$ is strictly related to $\ell_{\text {out }}$ by the relation $\gamma=\sqrt{\ell_{\text {out }} /\left(1-\ell_{\text {out }}\right)}$. The value of $\ell_{\text {out }}$, in turn, depends on the off-axis displacement of the input beam (Oemrawsingh et al. 2004):

$\ell_{\text {out }}=\ell_{0} \mathrm{e}^{-\rho^{2} / w_{0}^{2}}$

were, in our case, $\ell_{0}=1$.

By substituting in Eq. (A.1) the expressions of the L-G modes defined in Eq. (1) we can analytically derive a general expression of the intensity distribution of the first order OV:

$I_{\text {out }}(r, \theta) \propto \exp \left(-\frac{2 r^{2}}{w^{2}}\right)\left(1+\frac{2 \sqrt{2} \gamma}{w} r \cos \theta+\frac{2 \gamma^{2}}{w^{2}} r^{2}\right)$

where $w$ is related to the Gaussian parameter $w_{0}$ through relation $w=w_{0} \sqrt{2\left(1+z^{\prime 2} / k^{2} w_{0}^{4}\right)}$. The angular coordinate of the phase singularity (the light minimum) must be $\theta_{0}=\pi$ and its radial coordinate $r_{0}$ is found by imposing the condition $I_{\text {out }}(r, \pi)=0$. The condition is fulfilled when $r_{0}=w / \sqrt{2} \gamma$, which means that the radial position of the phase singularity increases as $\gamma$ decreases. In other words, the central dark region migrates progressively away from the diffraction axis $z^{\prime}$, causing the pattern of the OV to loose the axial symmetry. As a result, the two opposite intensity maxima located along the direction $\theta=\pi$ present different values. The ratio between them can be evaluated as:

$R=\exp \left(-\frac{\sqrt{1+4 \gamma^{2}}}{4 \gamma^{2}}\right)\left(\frac{\sqrt{1+4 \gamma^{2}}-1}{\sqrt{1+4 \gamma^{2}}+1}\right)^{2}$ 


\section{References}

Allen, L., Beijersbergen, M. W., Spreeuw, R. J. C., \& Woerdman, J. P. 1992, Phys. Rev. A, 45, 8185

Arecchi, F. T., Giacomelli, G., Ramazza, P. L., \& Residori, S. 1991, Phys. Rev. Lett., 67, 3749

Barbieri, C., Dravins, D., Occhipinti, T., et al. 2007, J. Mod. Opt., 54, 191

Bazhenov, V. Y., Vasnetsov, M. V., \& Soskin, M. S. 1990, JETP Lett., 52, 429

Beijersbergen, M. W., Coerwinkel, R. P. C., Kristensen, M., \& Woerdman, J. P. 1994, Opt. Commun., 112, 321

Bekshaev, A. Y., \& Karamoch, A. I. 2008, Opt. Commun., 281, 1366

Clifford, M. A., Arlt, J., Courtial, J., \& Dholakia, K. 1998, Opt. Commun., 156, 300

Coullet, P., Gil, L., \& Rocca, F. 1989, Opt. Commun., 73, 403

Foo, G., Palacios, D. M., \& Swartzlander, Jr., G. A. 2005, Opt. Lett., 30, 3308

Fried, D. L. 1978, J. Opt. Soc. Am., 68, 1651

Grier, D. G. 2003, Nature, 424, 810

Halbwachs, J. L. 1981, A\&AS, 44, 47

Harwit, M. 2003, ApJ, 597, 1266

Jenkins, C. 2008, MNRAS, 384, 515

King, I. R. 1971, PASP, 83, 199

Law, N. M., Hodgkin, S. T., \& Mackay, C. D. 2006a, MNRAS, 368, 1917

Law, N. M., Mackay, C. D., \& Baldwin, J. E. 2006b, A\&A, 446, 739

Leach, J., \& Padgett, M. 2003, New J. Phys., 5, 154
Lee, J. H., Foo, G., Johnson, E. G., \& Swartzlander, Jr., G. A. 2006, Phys. Rev. Lett., 97, 053901

Mawet, D., Riaud, P., Absil, O., \& Surdej, J. 2005, ApJ, 633, 1191

McAlister, H. A., Hartkopf, W. I., Sowell, J. R., Dombrowski, E. G., \& Franz, O. G. 1989, AJ, 97, 510

Molina-Terriza, G., Torres, J. P., \& Torner, L. 2007, Nature Phys., 3, 305

Nye, J. F., \& Berry, M. V. 1974, Proc. R. Soc. London A, 336, 165

Oemrawsingh, S. S. R., Eliel, E. R., Nienhuis, G., \& Woerdman, J. P. 2004, J. Opt. Soc. Am. A, 21, 2089

Padgett, M. J., \& Allen, L. 1995, Opt. Commun., 121, 36

Palacios, D. M., Maleev, I. D., Marathay, A. S., \& Swartzlander, Jr., G. A. 2004, Phys. Rev. Lett., 92, 143905

Shvedov, V., Krolikowski, W., Volyar, A., et al. 2005, Opt. Expr., 13, 7393

Swartzlander, Jr., G. A. 2001, Opt. Lett., 26, 497

Swartzlander, Jr., G. A. 2006, Opt. Lett., 31, 2042

Swartzlander, Jr., G. A., \& Law, C. T. 1992, Phys. Rev. Lett., 69, 2503

Swartzlander, Jr., G. A., Ford, E. L., Abdul-Malik, R. S., et al. 2008, Opt. Expr., 16,10200

Tamburini, F., Anzolin, G., Umbriaco, G., Bianchini, A., \& Barbieri, C. 2006a, Phys. Rev. Lett., 97, 163903

Tamburini, F., Umbriaco, G., Anzolin, G., Barbieri, C., \& Bianchini, A. 2006b, Mem. Soc. Astron. It. Suppl., 9, 484

Thidé, B., Then, H., Sjöholm, J., et al. 2007, Phys. Rev. Lett., 99, 087701

Vaziri, A., Weihs, G., \& Zeilinger, A. 2002, J. Opt. B, 4, 47 